\title{
Synchronous Onset of Breast and Pancreatic Cancers: Results of Germline and Somatic Genetic Analysis
}

\author{
Michael Castro ${ }^{a} \quad K^{\prime}$ ah Vierkoetter ${ }^{b} \quad$ Douglas Prager ${ }^{c}$ \\ Shasta Montgomery ${ }^{a}$ Kristin Sedgwick $^{d}$ \\ ${ }^{a}$ Personalized Cancer Medicine, PLLC, Honolulu, Hawaii, USA; ${ }^{\circ}$ Department of Pathology, \\ Queens Medical Center, Honolulu, Hawaii, USA; ${ }^{C}$ Department of Radiology, Queens \\ Medical Center, Honolulu, Hawaii, USA; ${ }^{d}$ Counsyl Inc., San Francisco, Calif., USA
}

\section{Keywords}

BRCA2 - Hybrid capture - Next-generation sequencing - Breast cancer - Pancreas cancer - FAT1 - CREBBP $\cdot$ Health policy

\begin{abstract}
Background: Synchronous cancers have occasionally been detected at initial diagnosis among patients with breast and ovarian cancer. However, simultaneous coexistence and diagnosis of breast and pancreas cancer has not previously been reported. Case Report: Paternal transmission of a germline BRCA2 mutation to a patient who was diagnosed at age 40 with locally advanced breast and pancreas cancer is presented. Somatic genomic analysis of both cancers with next-generation DNA sequencing confirmed the germline result and reported a variety of variants of unknown significance alterations, of which two were present in both the breast and pancreas cancers. Discussion: The possibility that genomic alterations could have been responsible for modulating the phenotypic or clinical expression of this rare presentation is considered. The authors call attention to the practice of privatizing the clinicogenetic information gained from genetic testing and call for health policy that will facilitate sharing in order to advance the outcomes of patients diagnosed with hereditary cancers.
\end{abstract}




\section{Case Reports in Oncology}

\section{Background}

Since the discovery of the tumor suppressor genes BRCA1 and BRCA2 in 1997, it is widely recognized that carriers of germline mutations have an elevated risk of developing breast and ovarian cancer by autosomal dominant transmission. From an array of epidemiologic studies, the burden of cancer associated with these mutations also includes a heightened risk for a variety of other malignancies, including prostate cancer, pancreatic cancer, melanoma and other skin cancers, stomach and colorectal cancer [1] (table 1). Subsequently, recommendations [2] evolved to recommend prostate cancer screening in men harboring BRCA mutations [3], as well as abdominal MRI and early endoscopic screening of BRCA patients whose families include a diagnosis of pancreas [4] or colorectal cancer [5], respectively.

Following a diagnosis of breast cancer, the risk of a second breast cancer approaches $27 \%$ within 5 years. However, simultaneous diagnoses of multisite cancer with contralateral breast cancer and coexistent breast and ovarian cancer have been reported at initial diagnosis $[6,7]$. We report here the case of a woman with a germline BRCA2 mutation who presented with synchronous onset of breast cancer and pancreatic cancer.

\section{Case Report}

The patient is a 41-year-old premenopausal woman who discovered a lump in the upper outer quadrant of the left breast. The workup was conducted in July 2015 within a week of symptom onset. Bilateral diagnostic mammography revealed pleomorphic calcifications in the area of concern. An ultrasound revealed a $3.2-\mathrm{cm}$ solid mass in the 2:00 position $8 \mathrm{~cm}$ from the nipple, and 2 smaller masses in the $2: 30$ position that were $4 \mathrm{~cm}$ from the nipple, which appeared to represent multifocal disease. An ultrasound-guided breast biopsy was performed. The 2:00 o'clock lesion contained infiltrating duct carcinoma with intermediateto high-grade features and duct carcinoma in situ (DCIS) with comedonecrosis. The 2:30 lesion contained DCIS as well. The estrogen receptor (ER) stained $100 \%$, and the progesterone receptor $12 \%$. The HER2 FISH was 1.1. An MRI of the breast was performed which showed a suspicious lesion on the right side. A biopsy of the contralateral breast lesion was negative. The left axillary lymph nodes (LNs) were sampled by ultrasound-guided fine needle aspiration and were negative.

Because the breast cancer was locally advanced by examination, a metastatic survey was undertaken. Metastatic disease was not discovered; however, a CT of the body (fig. 1) revealed a 2.6-cm mass in the tail of the pancreas. The CA19-9 was 126 (normal 0-38). A positron emission tomography (PET) scan was positive in the pancreas with an SUV level of 3.6. A CT-guided biopsy of the lesion revealed adenocarcinoma. Immunostains for villin and CA19-9 were positive, and negative for ER, establishing pancreatic origin and ruling out the diagnosis of a metastatic lesion (fig. 2).

The patient was taken to the operating room in August 2015 and underwent a left modified radical mastectomy. Pathology revealed a 7.5-cm grade 2 (tubule 3, nuclear 3, mitoses 1 $=7 / 9$ ) infiltrating duct carcinoma with lymphovascular invasion. A component of high-grade DCIS with solid and cribriform features occupying 50-60\% of the tumor and containing comedonecrosis was also identified. The deep margins for both the invasive and in situ components were clear by $0.6 \mathrm{~cm}$ and $8 / 23 \mathrm{LNs}$ were found to contain metastatic cancer. She was considered to have a G2 pT3N2aM0, AJCC stage IIIA breast cancer. 


\section{Case Reports in Oncology}

In September 2015, the patient underwent a laparoscopic, hand-assisted splenectomy, distal pancreatectomy, lymphadenectomy, and concomitant bilateral salpingooophorectomy. Pathology revealed a $3.2 \times 2.3 \times 2.0 \mathrm{~cm}$ moderately differentiated adenocarcinoma with invasion of the peripancreatic fat. Perineural invasion was present, but vascular invasion was not. The resection margins were $1.8 \mathrm{~cm}$ from proximal pancreatic margin; 1/13 regional LNs contained metastatic disease. She was considered to have G2, pT2N1M0, AJCC stage IIB pancreatic cancer. The fallopian tubes and ovaries were submitted entirely for microscopic examination and were histologically unremarkable.

Germline genetic testing (Inherited Cancer Screen, Counsyl Inc., South San Francisco, Calif., USA) confirmed the presence of a deleterious BRCA2 mutation characterized as c.5681(dupA). Her father, who was diagnosed with prostate cancer at age 69, and her younger brother carry the same BRCA2 mutation. No mutations were identified in 23 other genes associated with hereditary cancer [8].

Genomic profiling was performed on both cancers (FoundationOne ${ }^{\mathrm{TM}}$, Foundation Medicine, Inc., Cambridge, Mass., USA) employing hybrid capture, next-generation sequencing of DNA in the exomic regions of 315 genes. The results of this somatic analysis revealed the mutation Y1894fs*1, also known as c.5681(dupA) in both cancers, as well as a second BRCA2 mutation and a KRAS G12R mutation in the pancreatic cancer. Common genetic alterations of unknown significance in FAT1 and CREBBP genes were also identified in both the breast and pancreas cancers (table 2).

The patient received genetic counseling. Adjuvant chemotherapy utilizing doxorubicincisplatin followed by gemcitabine-NAB paclitaxel was administered postoperatively. The patient will receive postmastectomy radiotherapy, upper abdominal radiotherapy, aromatase inhibitor therapy, and will be considered for olaparib therapy in view of her still ominous prognosis, despite the fact that she could not qualify for participation in a clinical trial with olaparib because of the exclusion of patients with two primary malignancies.

\section{Family History}

The pedigree reveals a small kindred. The patient's father was diagnosed with prostate cancer at age 69. He was confirmed as carrying the same BRCA2 mutation as his daughter. The patient's brother, who is a decade younger, also carries the familial mutation, but so far has not been diagnosed with a malignancy at age 30 . The paternal grandfather had lung cancer (fig. 3).

\section{Discussion}

The patient presented with locally advanced breast cancer. A search for metastatic disease led to the diagnosis of pancreatic cancer before the development of overt distant metastatic spread, creating the possibility that she might be cured of both cancers with multimodality therapy. While it is commonplace that physicians caring for breast cancer patients with BRCA mutations consider the possibility of a second cancer in the form of contralateral breast or ovarian cancer at diagnosis, our experience with this patient suggests that clinical suspicion about other BRCA-related cancers is needed. Though scanning for metastatic disease in the adjuvant breast has a low yield for discovering metastatic disease among earlystage patients, the possibility of a second primary malignancy in a young patient with a BRCA-related malignancy introduces the question and clinical responsibility pertaining to scanning for second cancers. On the other hand, the presentation with more than one prima- 


\section{Case Reports in Oncology}

ry cancer is uncommon. To our knowledge, simultaneous onset of both breast and pancreatic cancer has never been reported in the BRCA literature or any other case report before.

The differential diagnosis that the patient might have a single cancer with metastatic disease was considered in the pathologic evaluation. However, immunohistochemical staining revealed distinctly different protein expression patterns with regards to ER (100 vs. $0 \%$ in the breast and pancreas tumors, respectively). While villin expression is only found in approximately $30 \%$ of pancreatic cancers, its presence here supports the impression of two distinct primary cancers. The molecular profiling results also show that the two cancers were different, including the finding of KRAS mutation in the pancreas cancer, characteristic of that malignancy, but only found in approximately $5 \%$ of breast cancers and not seen in this patient's breast primary.

The somatic genomic analysis of both cancers were remarkable for showing that two additional alterations or variants of unknown significance (VUS) were observed in known drivers of malignancy involving the FAT1 and CREBBP1 genes that were present in both cancers. FAT1 is a tumor suppressor gene involving the $\mathrm{WNT} / \beta$-catenin pathway in a wide variety of cancers, including but not limited to pancreatic cancer, glioblastoma, head and neck cancer, colorectal cancer, and ovarian cancer [8]. FAT1 inhibits $\beta$-catenin nuclear translocation and transcriptional activity, and mutation leads to upregulation of WNT/ $\beta$-catenin signaling involved in driving proliferation by disrupting cadherin domains involved in cell adhesion. Additionally, FAT1 overexpression has been associated with diminished survival in at least one data set [9]. CREBBP is involved in chromatin remodeling and acetylation, and suggests that this patient's cancers could share epigenetic dysregulation as a common pathogenic feature. That identical gene alterations were found in two genes common in both cancers suggests they may represent coexistent drivers of malignant behavior. Conceivably, they could have been involved in propelling the rare phenotype of two synchronous cancers. The somatic analysis of this patient's cancers complements the germline hereditary cancer diagnosis and raises the question whether the expression of BRCA2 mutation can be modulated by other genomic factors. Whether these particular VUS alterations played a role in the phenotypic expression of the underlying BRCA2 mutation will require further clinicogenomic correlations in the future. Additionally, it would be of interest to study the father's prostate cancer to see if the same abnormalities are present in his cancer.

The germline BRCA2 mutation c.5681(dupA) carried by this patient has rarely been identified in the publicly maintained databases and not previously been reported in association with prostate or pancreatic cancer or multiple primary malignancies. Nevertheless, this particular mutation has been identified in other reported cases of hereditary breast and ovarian cancer [10]. It results in a frameshift variant and is presumed deleterious [11]. However, the largest database in existence is closed to inquiry. Because clinicogenetic correlations such as this could have life-saving value in advising mutation carriers with regard to risk, optimal screening strategies, and preventive surgery, the authors call into question the practice of maintaining 'proprietary' databases where knowledge that might have been shared for enhancing clinical outcomes of early diagnosis, prevention, and improved survival is instead privatized and exploited for profit.

\section{Conclusion}

This case report portrays the simultaneous diagnosis of breast and pancreatic cancers in a patient with an uncommon BRCA2 mutation. The identification of other DNA alterations by 
profiling the somatic tumor genome uncovered alterations in two known drivers of malignancy, FAT1 and CREBBP, leading the authors to speculate about other factors in the intracellular milieu that might have led to this rare presentation.

Undoubtedly, much insight will come from combining analyses of germline and somatic mutations with clinical presentation and patient outcomes in the future. But this will depend upon sharing the responsibility for curation and clinicogenomic correlation. Health policy that discourages privatization of genetic results would be of value in advancing our knowledge of genetic predisposition and disease behavior, and enhance the use of that knowledge for the protection of existing and future patients.

\section{Statement of Ethics}

The patient provided written consent for her case to be published.

\section{Disclosure Statement}

Kristin Sedgwick is a genetic counselor employed at Counsyl Inc., a molecular diagnostics laboratory. The remaining authors disclose no conflicts of interest.

\section{References}

1 Friedenson B: BRCA1 and BRCA2 pathways and the risk of cancers other than breast or ovarian. Med Gen Med 2005;7:60.

2 Burke W, Daly M, Garber J, Botkin J, Kahn MJ, Lynch P, et al: Recommendations for follow-up care of individuals with an inherited predisposition to cancer. II. BRCA1 and BRCA2. Cancer Genetics Studies Consortium. JAMA 1997;277:997-1003.

3 National Comprehensive Cancer Network (NCCN): NCCN Clinical practice guidelines in oncology. http://www.nccn.org/professionals/physician_gls/f_guidelines.asp.

-4 Canto MI, Harinck F, Hruban RH, Offerhaus GJ, Poley JW, Kamel I, Nio Y, Schulick RS, Bassi C, Kluijt I, Levy MJ, Chak A, Fockens P, Goggins M, Bruno M; on behalf of the International Cancer of the Pancreas Screening (CAPS) Consortium. International Cancer of the Pancreas Screening (CAPS) Consortium summit on the management of patients with increased risk for familial pancreatic cancer. Gut 2013;62:339-347.

$5 \quad$ NCCN Clinical Practice Guidelines in Oncology. Colorectal cancer screening, version 1. 2014. http://www.nccn.org/professionals/physician_gls/pdf/colorectal_screening.pdf.

-6 Dilawari A, Cangiarella J, Smith J, Huang A, Downey A, Muggia F: Co-existence of breast and ovarian cancers in BRCA germ-line mutation carriers. Ecancermedicalscience 2008;2:109.

-7 Mavaddat N, Peock S, Frost D, Ellis S, Platte R, Fineberg E, et al: Cancer risks forBRCA1 and BRCA2 mutation carriers: results from prospective analysis of EMBRACE. J Natl Cancer Inst 2013;105:812822.

8 Counsyl gene panel for hereditary cancer: https://www.counsyl.com/services/inherited-cancerscreen/conditions/.

-9 Morris LG, Kaufman AM, Gong Y, Ramaswami D, Walsh LA, Turcan S, Eng S, et al: Recurrent somatic mutation of FAT1 in multiple human cancers leads to aberrant Wnt activation. Nature Genetics 2013;45:253-261.

10 Jianmin Wu: http://www.pancreaticcancer.net.au/news/files/fat1-cadherin-as-aserum-markerinpancreatic-cancer.

11 ClinVar database: http://www.ncbi.nlm.nih.gov/clinvar/variation/37988/. 


\section{Case Reports in Oncology}

\begin{tabular}{l|l}
\hline Case Rep Oncol 2016;9:387-394 \\
\hline $10.1159 / 000447348$ & $\begin{array}{l}\text { C } 2016 \text { The Author(s). Published by S. Karger AG, Basel } \\
\text { www.karger.com/cro }\end{array}$ \\
\hline
\end{tabular}

Castro et al:: Synchronous Onset of Breast and Pancreatic Cancers: Results of Germline and Somatic Genetic Analysis
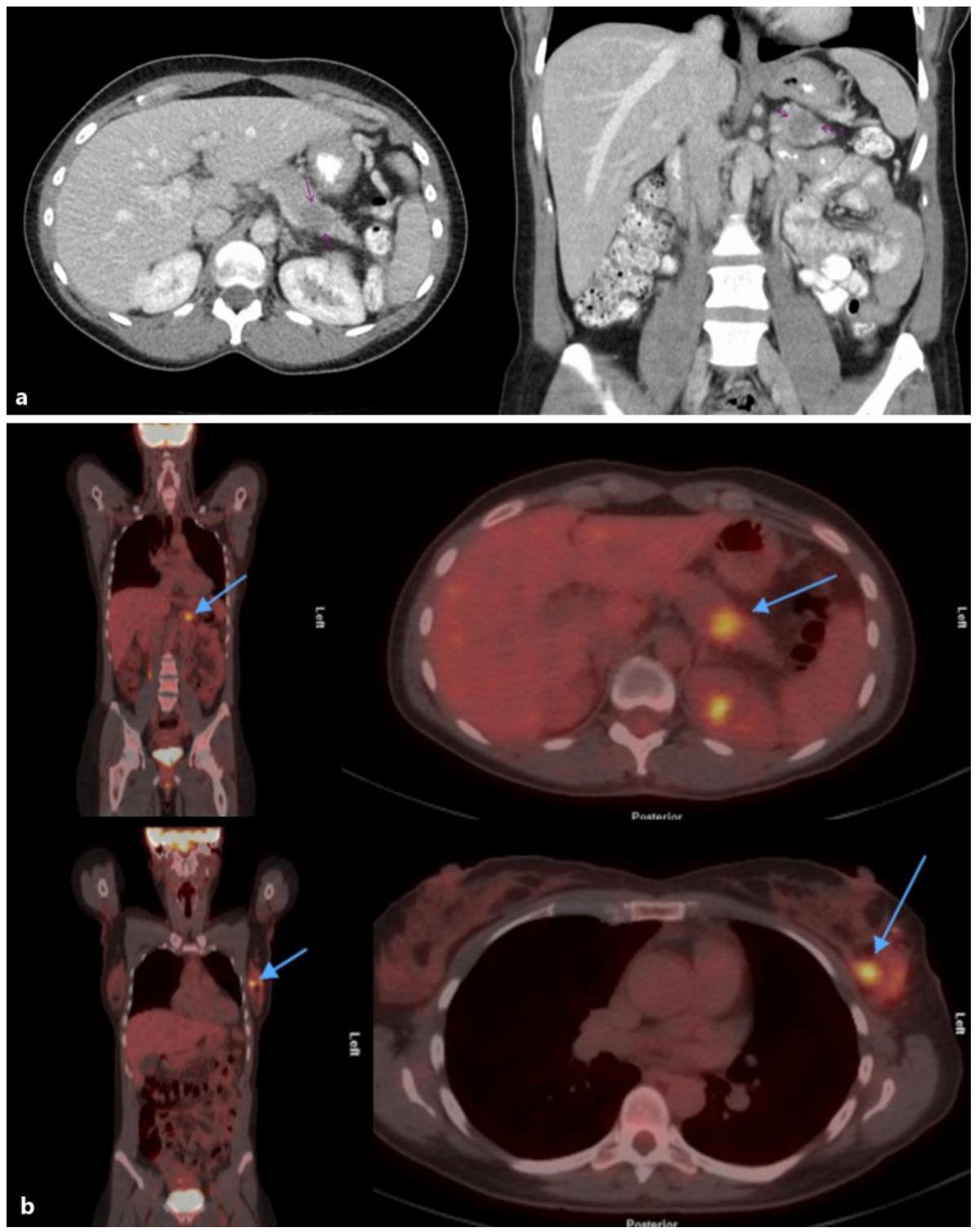

Fig. 1. CT and FDG-PET/CT scans obtained at diagnosis. a A neoplastic lesion was identified in the tail of the pancreas. b Hypermetabolism is identified in the tail of pancreas lesion and axillary LNs. Primary breast lesion is not shown. 


\section{Case Reports in Oncology}

\begin{tabular}{l|l}
\hline Case Rep Oncol 2016;9:387-394 \\
\hline $10.1159 / 000447348$ & $\begin{array}{l}\text { (c) 2016 The Author(s). Published by S. Karger AG, Basel } \\
\text { www.karger.com/cro }\end{array}$ \\
\hline
\end{tabular}

Castro et al.: Synchronous Onset of Breast and Pancreatic Cancers: Results of Germline and Somatic Genetic Analysis

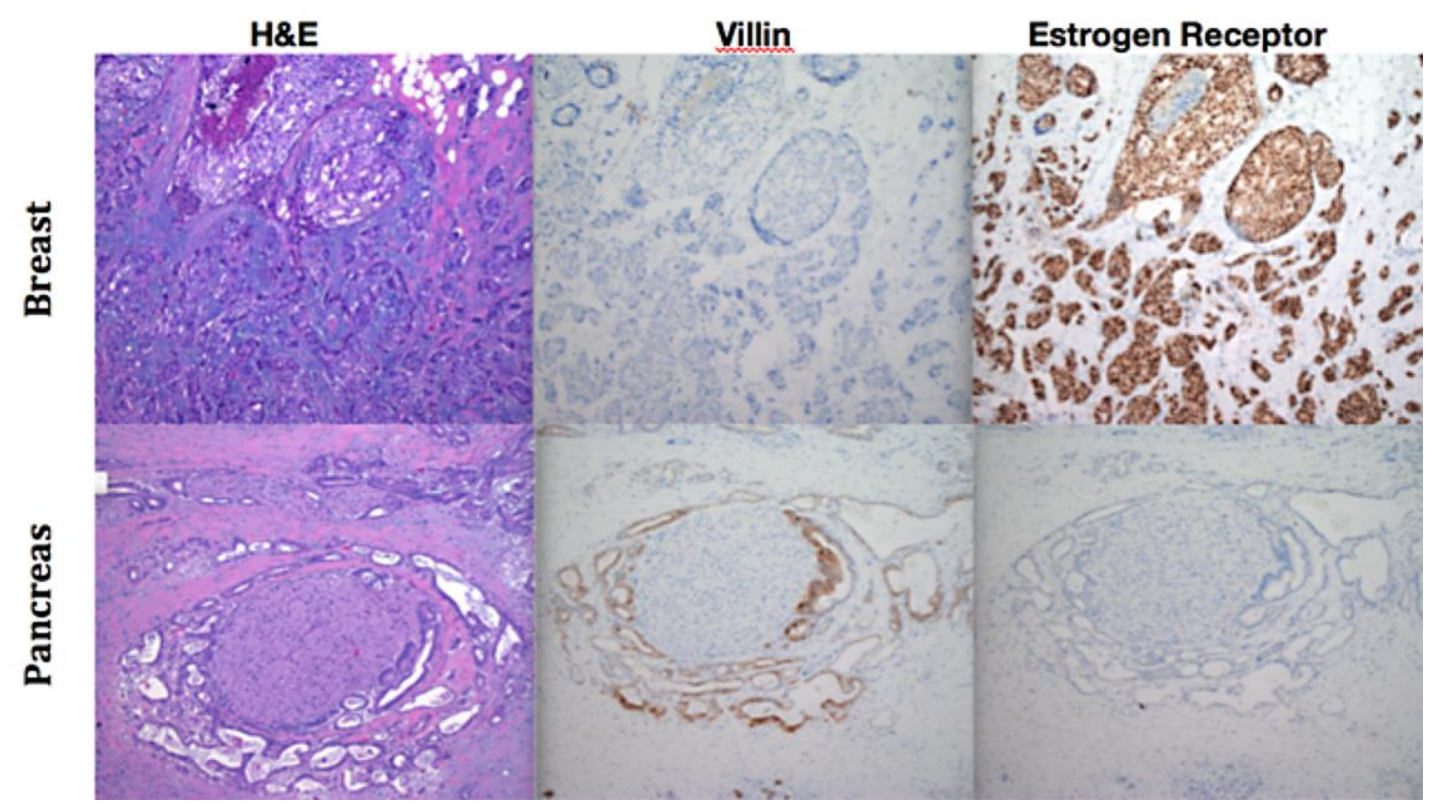

Fig. 2. Histology and immunostains of breast and pancreas cancers diagnosed at presentation. Top panels: breast cancer, bottom panels: pancreas cancer. Left column: hematoxylin and eosin stains, middle column: villin stains, right column: ER stains.

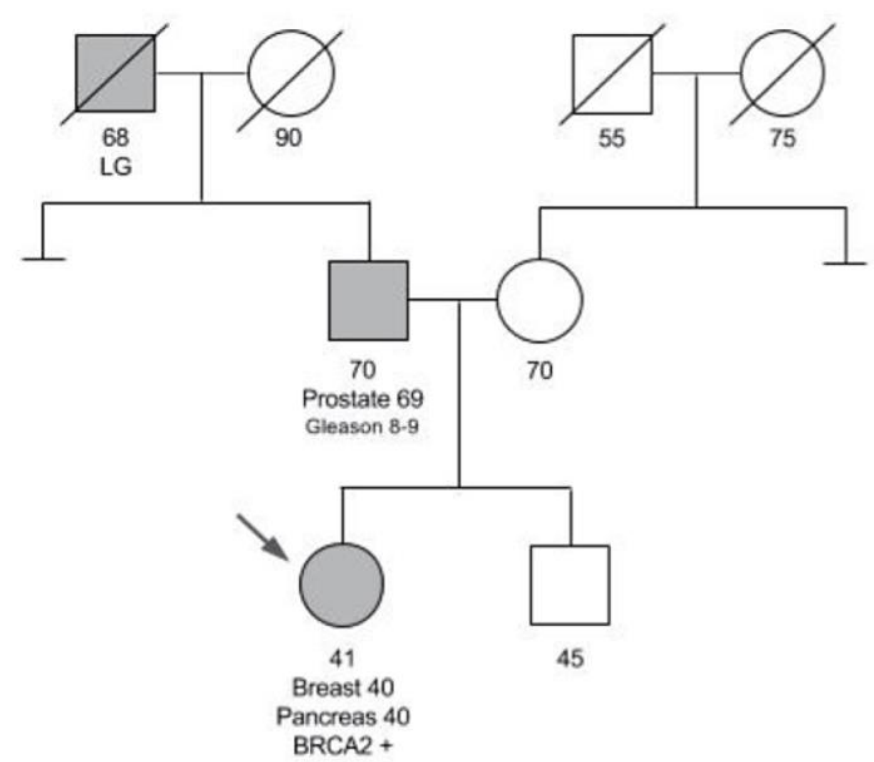

Fig. 3. Pedigree of the patient. Anonymized pedigree of a 41-year-old woman diagnosed with synchronous cancers of the breast and pancreas and found to harbor a deleterious mutation in the BRCA2 gene. Shading represents affected individuals. 
Table 1. The lifetime risk for these cancers in individuals with a pathogenic variant in BRCA1 or BRCA

\begin{tabular}{lcc}
\hline Cancer site & BRCA1 & BRCA2 \\
\hline Breast cancer & $50-80 \%$ & $40-70 \%$ \\
2nd breast cancer & $27 \%$ within 5 years & $12 \%$ within 5 years, \\
& & $40-50 \%$ at 20 years \\
Ovarian cancer & $24-40 \%$ & $11-18 \%$ \\
Male breast cancer & $1-2 \%$ & $5-10 \%$ \\
Prostate cancer & $<30 \%$ & $<39 \%$ \\
Pancreatic cancer & $1-3 \%$ & $2-7 \%$ \\
\hline
\end{tabular}

Table 2. Genomic profiling results of breast and pancreas cancers (Foundation Medicine Inc.)

\begin{tabular}{ll}
\hline Breast cancer & Pancreatic cancer \\
\hline BRCA2* & BRCA2* \\
Y1894fs*1 & E2850fs*12, Y1894fs*1 \\
\hline VUS & KRAS \\
BLM & G12R \\
amplification & BCOR \\
\hline GATA3 & L1514Q \\
amplification & FLT4 \\
\hline CHD2 & A644S \\
amplification & DNMT3A \\
\hline IDH2 & C562Y \\
amplification & MLL \\
\hline MLL & L3614P \\
L3614P & CREBBP* \\
\hline CREBBP* & M747V \\
M747V & MLL2 \\
\hline IGF1R & P2210L \\
amplification & TGFBR2 \\
\hline SMAD3 & splice site 95 - 28_165del99 \\
amplification & FAT1* \\
\hline FAT1* & R4208W \\
R4208W & \\
\hline NTRK3 & \\
amplification & \\
\hline MAP2K1 & \\
amplification & \\
\hline
\end{tabular}

In addition to the mutation in BRCA2, common alterations were observed in CREBBP and FAT1 genes. Mutation sites common in both cancers are denoted with an asterisk. 